\title{
Synergy between trastuzumab and pertuzumab for human epidermal growth factor 2 (Her2) from colocalization: an in silico based mechanism
}

\author{
Gloria Fuentes ${ }^{1 *}$, Maurizio Scaltriti ${ }^{2,3}$, José Baselga ${ }^{2,3}$ and Chandra S Verma ${ }^{1,4,5^{*}}$
}

\begin{abstract}
Introduction: Human epidermal growth factor 2 (Her2), a receptor tyrosine kinase, is overexpressed in breast cancers. It has been successfully targeted by small molecule kinase inhibitors and by antibodies. Recent clinical data show a synergistic response in patients when two antibodies, trastuzumab and pertuzumab, are given in combination.
\end{abstract}

Methods: This unexpected effect is rationalized through computer models and molecular dynamic simulations by hypothesizing that the two antibodies can co-localize on the same molecule of the Her2 extracellular domain.

Results: Simulations suggest that the clinical synergism observed for the two antibodies arises partly from enhanced affinity that originates in cooperative interactions between these two antibodies when they are colocalized on Her2 and "clamp" it; this may inhibit dimerization and possibly higher oligomerizations with neighboring receptors. In the presence of trastuzumab, the receptor becomes highly plastic, especially domains I to III, and this appears to promote increased association with pertuzumab. Further, the presence of pertuzumab evokes novel interactions between the receptor and trastuzumab. Indeed, splicing out of this region in silico results in a big reduction in the interactions of the antibody with the receptor.

Conclusions: If validated, these findings will bring about a new direction in the design of antibodies whereby different epitopes on the same antibody may be targeted to lead to synergistic/cooperative inhibition and contribute to generate more potent therapeutics and to increase clinical efficacy.

\section{Introduction}

ErbB receptors are the prototypical founders of the growth factor receptor tyrosine kinase (RTK) family. They are activated by the binding of different ligands and are involved in the transmission of signals from the extracellular space to the cytoplasm and nucleus of a cell, thus orchestrating biological processes [1]. Among the members of this family, which also includes the epidermal growth factor receptor (EGFR or ErbB1), ErbB3, ErbB4, Her2 or ErbB2 is homologous to, but distinct from the others, since it is not activated upon ligand binding. This ligand-independent activation makes Her2 the universal heterodimeric partner for each of the other ErbB family members [2]. The architecture of

\footnotetext{
* Correspondence: gfuentes@bii.a-star.edu.sg; chandra@bii.a-star.edu.sg 'Bioinformatics Institute (A*STAR), 30 Biopolis Street, \#07-01 Matrix, 38671 , Singapore

Full list of author information is available at the end of the article
}

these receptors $[3,4]$ reveals an extracellular domain (made up of four subdomains), a single transmembrane helix, and an intracellular domain (consisting of a juxtamembrane region, a tyrosine kinase domain, and a Cterminal tail harboring autophosphorylation sites that serve as docking sites for adaptor proteins [5,6]). The importance of the tight regulation of these receptors and in particular Her2 is signified in human breast cancers, where Her2 is overexpressed by approximately 20 to $30 \%$ and this is normally associated with more aggressive tumours and a poorer prognosis $[7,8]$. Due to this active role in human cancers, a number of therapeutic approaches are currently under development to block the effects of Her2 overexpression, including kinase inhibitors (such as lapatinib) and monoclonal antibodies (trastuzumab and pertuzumab) [9]. The antiHer2 monoclonal antibody trastuzumab binds to domain IV of Her2, a region that is not involved in 
receptor dimerization and is thought to work in a complex manner [10,11]. In contrast, pertuzumab binds to Her2 near the centre of the domain II dimerization arm (Figure 1). The overlap between the pertuzumab epitope and the probable heterodimer interface suggests steric occlusion by physically blocking the formation of Her2containing heterodimers. Recent clinical observations have demonstrated that combining trastuzumab and pertuzumab together yields surprisingly synergistic results in tumour inhibition (that is, the combined effect of using the two molecules was far greater in effect than when using either alone) during the treatment of Her2 positive breast cancers that progressed during prior trastuzumab therapy [12]. This suggests a cooperative mechanism of inhibition that can lead to clinical improvement in the treatment of these tumours.

We have used molecular modelling to develop a mechanism that may partly explain this cooperative effect. A simple model is that cooperativity could arise from the physical co-localization of the two antibodies on the same Her 2 molecule, as has been previously demonstrated for the insulin-like growth factor receptor [13]. Models of the binary structures of Her2:trastuzumab and Her2:pertuzumab were combined to create a model of the ternary complex of Her 2 and the two Fab fragments of these antibodies. This system was subjected to structural/energetic refinement followed by molecular dynamics (MD) simulations (Additional file 1).

\section{Methods}

\section{Building the models}

The binary associations of Her2 and the two antibodies have been experimentally characterized and are available in the Protein Data Base (pdb entries: 1n8z[3] and 1 s78 [14]). The electronic density of the C-terminal region of the receptor is the Her2:pertuzumab complex is missing, due probably to the high inherent flexibility of this part of the structure. For the coordinates of the residues in this region, we have assumed that this stretch will adopt a conformation similar to the one found in the complex with trastuzumab and have been so modeled. A few other missing residues, located in loops, have been modeled based on homologue structures. The ternary model of the complex Her2 and the two Fab domains of these antibodies, trastuzumab and pertuzumab, were manually constructed using the coordinates of the previous models, after superposition on the backbone of the receptor ectodomain.

\section{MD simulations and binding energy calculations}

We have used seven different systems for this study (Additional file 1); the complete 3D model structure of the complex (Her2-P-T), the receptor bound to trastuzumab (Her2-T) and pertuzumab (Her2-P), the apo- receptor (Her2), the two independent antibodies (pert and trast) and both antibodies co-localized as they are found in the complex (Abs). All these systems have been refined using several energy minimization and short MD simulation cycles in order to equilibrate the structures after which they were subjected to the production phases of MD simulation (around 18 to $20 \mathrm{~ns}$ each) in explicit water, as detailed. The AMBER (Assisted Model Building with Energy Refinement) 9 package [15] together with the ff99 force field [16] was used. The protonation states of the residues were assigned using PDB2PQR [17], after which the systems were surrounded with a box of TIP3 or Transferrable Interaction Potential 3 water molecules that result in an $8 \AA$ layer of water molecules around the proteins and neutralized by the necessary numbers of $\mathrm{Cl}$-or $\mathrm{Na}+$ counter-ions using the t-leap module of AMBER 9. The solvated systems were then subjected to a minimization phase, mainly by the steepest descend method and small number of conjugate gradient steps, in order to remove bad contacts. After that a 50 ps heating phase was carried out, where the proteins were heated from 0 to 300 $\mathrm{K}$, followed by another 50 ps phase density equilibration at a constant pressure of $1 \mathrm{~atm}$. A final 2 ns unconstrained MD simulation was carried out. The production runs were extended to around $20 \mathrm{~ns}$ at constant temperature using a Langevin thermostat [18] and constant pressure (1 atm) by coupling to a Berendsen barostat These two queries do not need to be address since these are mathematical methods and not devices [19]. The particle mesh Ewald (PME) method [20] was used with a direct-space non-bonded cutoff of $8 \AA$ to account for long range interactions. Covalent bond lengths to hydrogen atoms were constrained using the SHAKE algorithm [21], which allowed a time step of 2 fs for all the MD simulations. Snapshots in the interval of 8 to 16 ns were extracted for further processing. The analysis was carried out using functionalities in the ptraj module of AMBER 9, the module Bio3D [22] of $\mathrm{R}$ and Dyndom [23]. The PyMOL molecular viewer, a software package [24] was used for visualizing and generating the figures.

The binding free energy $\Delta G_{\text {bind }}$ of the different systems was calculated according to the so-called Molecular Mechanics/Generalized-Born Surface Area (MM/ GBSA) procedure [25]. Here $\Delta G_{\text {bind }}$ denotes the contributions from the molecular mechanics (MM) energies of the molecules, and originates in contributions from internal energies, electrostatics $\left(\Delta G_{E L E}\right)$ and van der Waals $\left(\Delta G_{V D W}\right)$ contributions. These terms have been calculated using the mm_pbsa module of AMBER 9. The polar component was evaluated with the Generalized-Born (GB) approach. In this approach, the nonpolar contribution to the solvation energy was calculated as $\Delta \mathrm{G}_{\mathrm{NP}}=\gamma(\mathrm{SASA})+\beta$, in which $\gamma=0.00542 \mathrm{kcal} / \AA$, $\beta$ 
$=0.92 \mathrm{kcal} / \mathrm{mol}$ and SASA is the solvent-accessible surface area estimated with LCPO (linear combination of pairwise overlaps) method [26] as implemented in AMBER. While the real free energy of a system also includes contributions from the entropies, given the large sizes of the systems studied here, computations of entropies were prohibitive and hence could not be carried out. However, the free energies computed here have been averaged over several conformations generated through MD simulations, and hence the effects of entropy to some extent are implicitly included in the current calculations.

\section{Essential dynamics analysis and Dyndom analysis of Her2 and its antibodies}

Essential dynamics $[27,28]$ have been widely used for filtering large scale concerted fluctuations from an ensemble of conformations, like those obtained from MD simulations [29]. The method is comparable to a principal component analysis, and it is based on the construction of the diagonalized covariance matrix containing the atomic fluctuations. We have performed a standard essential dynamics (or Principal Component Analysis, PCA) analysis in order to identify the most relevant motions occurring in the receptor in the different bound states considered on the MD simulations carried out in this study (Additional file 2); all the covariance matrices have been calculated taking the apo receptor as a reference structure. This yields a set of eigenvectors in a $3 \mathrm{~N}$-dimensional space, and eigenvalues, reflecting the magnitude of the fluctuations along the corresponding eigenvectors. Each eigenvector describing a collective motion can be represented by two structures, one at the maximum value, the other at the minimum value of the collective variable when the trajectory is projected onto it. These two structures thus generated for the first eigenvector of each trajectory were used as input for the program DynDom [23], using default parameters, in order to determine dynamic domains, hinge axes and hinge-bending regions.

\section{Results and discussion}

\section{Conformational space of Her2 in different bound forms}

Simulations show that the ectodomain of Her2 adopts various conformations, depending on its bound state. The binary states (when complexed to trastuzumab or pertuzumab) are more stable compared to the apo or the ternary complex (Additional file 3 ). This could stem from the high intrinsic flexibility of the apo receptor or/and the ternary complex. Although a complete understanding of the behaviour of such a receptor demands the fulllength structure, we believe that the models used here represent valid starting structures for the type of analyses carried out in this study. As expected, the presence of an antibody immobilizes its corresponding epitope
(Additional file 4). The largest fluctuations in the pertuzumab binding region (ranging from 240 to 300) and in the trastuzumab epitope (around 550 to 600) are seen in the apo state or in the Her2:trastuzumab or Her2:pertuzumab complexes respectively. Interestingly the dimerization arm, a $\beta$ hairpin in domain II that protrudes from the rest of Her2, together with the spatially adjacent regions, undergoes enhanced fluctuations when trastuzumab binds. This may reinforce the fact that the Her2: trastuzumab complex can actually promote the association of Her2 with other members of the ErbB family.

We have also investigated the flexibility of the antibodies and the stability of their binding interfaces with Her2. In general, the complementary determining regions (CDR) exhibit smaller fluctuations in comparison to the rest of the antibody; whereas regions far from the receptor's influence remain extremely flexible. In the ternary complex, the C-terminal half of the light chain of pertuzumab shows an increased flexibility, induced by the presence of trastuzumab (Additional file 4). Trastuzumab shows a more complicated pattern. When only trastuzumab is present in the complex, the whole antibody shows a high flexibility, with the exception of the CDR for the light chain. In the ternary complex, the light chain undergoes the largest fluctuations in the region interacting with the receptor binding epitope; however the most relevant difference is an attenuation in the mobility of the $\mathrm{N}$-terminal half of the heavy chain due to the proximity of one of the disulphide-bonded modules of domain II, which in turn shows lower flexibility (residues 310 to 325; see section below on new epitopes). We can conclude that the dynamics of pertuzumab seem to be regulated mainly by the binding to the receptor; while for trastuzumab, the co-binding of the second antibody has a significant effect. When the two antibodies are analysed in the absence of the receptor, trastuzumab per se seems more flexible than pertuzumab, which can be the origin of the more complex dynamics observed for the systems including this antibody. These intricate changes and regulation between the different components of the system reflect the importance of flexibility and could point to "allosteric" changes in explaining the in vivo synergistic effect.

\section{Uncovering new epitopes in Her2}

The analysis of the interactions between Her2 and the two antibodies reveals that the perturbation of the crystallographically observed interfacial region of Her2 is small during the simulations of the binary complexes (Additional file 5). However, the co-localization of the two Abs on Her2 surprisingly triggers the formation of additional and novel contacts of Her2 with trastuzumab; these lie outside of its epitopes (Figure 1). This $\beta$-hairpin loop region, termed the pertuzumab-induced 


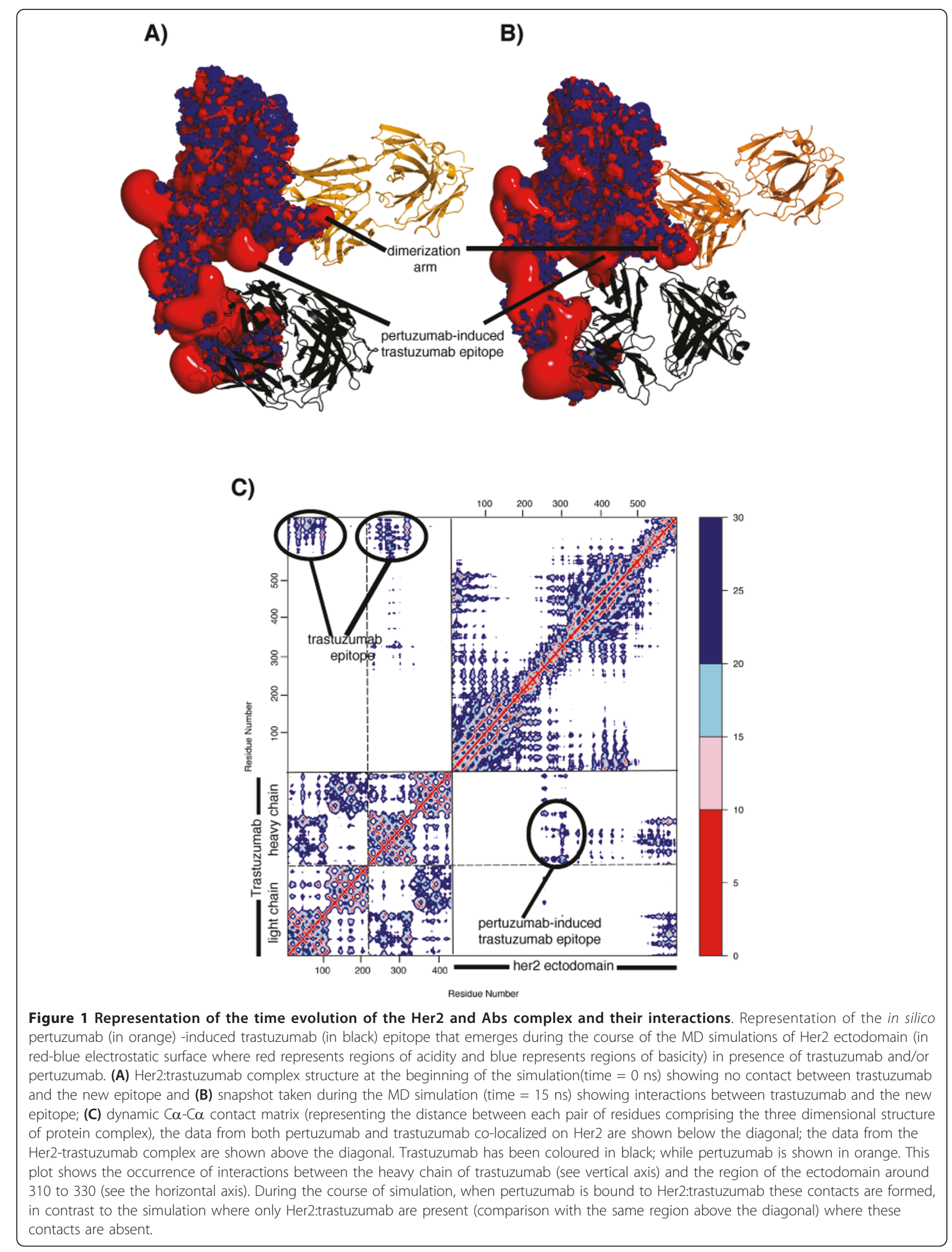


trastuzumab epitope, is located in domain II and is in proximity to the dimerization arm. The requirement of the co-localization of the two Abs for the formation of this new epitope is revealed in the minimum distance matrices for all the $\mathrm{C} \alpha$ atoms for the receptor and trastuzumab in the simulations of the binary and ternary complexes (see Figure 1C upper and lower diagonal of the matrix, respectively). This can also be observed from the reported distances between two residues in this novel epitope and residues at the interface in trastuzumab (Additional file 6). For this epitope to emerge in the Her2:trastuzumab interface, the presence of pertuzumab is strictly required. This novel association is mainly driven by long-range interactions, and could be the origin of the observed synergism.

\section{Quantification of synergism between pertuzumab and trastuzumab}

The MM-GBSA (molecular mechanics Generalized Born surface area) [25] is regularly employed to obtain estimates of the free binding energy of large systems and these are largely reasonable [30-32]. These only refer to the enthalpic component of the overall free energies of binding because computations of entropies of such large systems are prohibitively expensive. The derived association energetics of trastuzumab and pertuzumab to Her2 are shown in Table 1; the computed values are very large as is the case with computations involving large systems; indeed in comparison the binding of trastuzumab to the extracellular domain of Her2 and to Her2 positive cells ranges from approximately -12.5 to -14 $\mathrm{kcal} / \mathrm{mol}$ [33]. However, despite being large, qualitative analyses of the differences in the computed binding energies are very useful in ranking interactions. Our findings suggest (the more negative an energy, the more stable the associated system):

- trastuzumab has a higher affinity for apo Her2 than pertuzumab (Figure 2A); this arises from stronger electrostatic and van der Waals interactions between trastuzumab and Her2 (the net charge on Her2 is -15 , while on trastuzumab and pertuzumab it is +6 and +5 respectively); the buried area at the interface is approximately 1,675 and approximately $1,530 \AA^{2}$ for trastuzumab and pertuzumab, respectively

- a marked gain in affinity when both Abs are bound simultaneously to the receptor (synergism). This effect arises mainly from the interactions between these two antibodies upon co-localization, with a difference of approximately $27 \mathrm{kcal} / \mathrm{mol}$ between isolated and simultaneously bound antibodies (Figure 2C).

The computed energetics within these thermodynamic cycles suggest a sequential mechanism, where the initial binding involves trastuzumab (Figure 2A) followed by the binding of pertuzumab (Figure $2 \mathrm{~B}$ ). This seems to be partly driven by the conformational changes triggered upon the binding of trastuzumab that enhance pertuzumab binding (Figure 3) and the new additional contacts formed between trastuzumab and Her2. The enhanced interactions that accrue when the two antibodies are colocalized on Her2 leading to the cooperative effects, may provide a partial explanation for the clinically observed synergy. In order to support the effect of this newly revealed pertuzumab-induced trastuzumab epitope, we have constructed an in silico truncated form of Her2, where this $\beta$-hairpin has been deleted (T323C-AEDG$\mathrm{T} 328 \mathrm{C}$ ), and we find that the binding energetics undergo a marked destabilization (Table 1).

\section{Abs-induced bending of the Her2 ectodomain}

Principal Component Analysis (Additional file 2) combined with dynamical domain motion analysis using DynDom suggests that domains I to III (residues 1 to 480) generally behave as a rigid body, with hinge regions varying in their location in domain IV, depending on the binding partner of the receptor (Additional files 7 and 8). Two different motions are detected in the apo state of Her2; the first one involves a shearing motion between the stalk portion of the receptor and the large rigid-body unit; whereas the second one corresponds to a hinge motion within domain IV. The overall change in conformation involves a large conformational space scanned by the dimerization arm with respect to the membrane surface (with an angle

Table 1 In silico binding free energy for the association of Her2 ectodomain and the two antibodies

\begin{tabular}{|c|c|c|c|c|c|}
\hline & $\Delta \mathrm{G}_{\mathrm{ELE}}$ & $\Delta \mathrm{G}_{\mathrm{VDW}}$ & $\Delta \mathrm{G}_{\mathrm{GB}}$ & $\Delta \mathrm{G}_{\mathrm{NP}}$ & Total $\Delta \mathrm{G}$ \\
\hline Her2-T $<-H e r 2+$ trast & -841.6 & -116.3 & -197.8 & -14.8 & $-1,144.6$ \\
\hline Her2-P $<-$ Her2 + pert & -490.5 & -55 & -480.1 & -4.3 & $-1,095.7$ \\
\hline Her2-P-T $<-$ Her2 + trast + pert & $-1,028.8$ & -102.6 & -217.7 & -11.5 & $-1,438.8$ \\
\hline Her2-P-T $<$-Her2 + Abs & $-1,374.4$ & -114.9 & 730.4 & -17.6 & -851.0 \\
\hline Her2-trunc-P-T $<-$ Her2-trunc + trast + pert & $-1,044.3$ & -149.4 & 469.2 & -20 & -725.0 \\
\hline
\end{tabular}

Binding free energy components (kcal/mol) for the association of Her2 to pertuzumab and trastuzumab calculated using MM-GBSA method using MD snapshots extracted from independent simulations.

Her2-T, Her2-trastuzumab; trast: trastuzumab; Her2-P, Her2-pertuzumab; pert, pertuzumab; Her2-P-T, Her2-pertuzumab-trastuzumab; Abs, antibodies (here specifically it refers to both pertuzumab and trastuzumab); Her2-trunc, truncated form of Her2; $\Delta G_{\text {bind, }}$ binding free energy; MM/GBSA, molecular mechanics/ Generalized-Born surface Area; $\Delta \mathrm{G}_{\mathrm{ELE}}$, electrostatics energy; $\Delta \mathrm{G}_{\mathrm{VDW}}$, van der Waals energy; $\mathrm{GB}$, Generalized-Born; $\Delta \mathrm{G}_{\mathrm{NP}}$, non-polar energy 


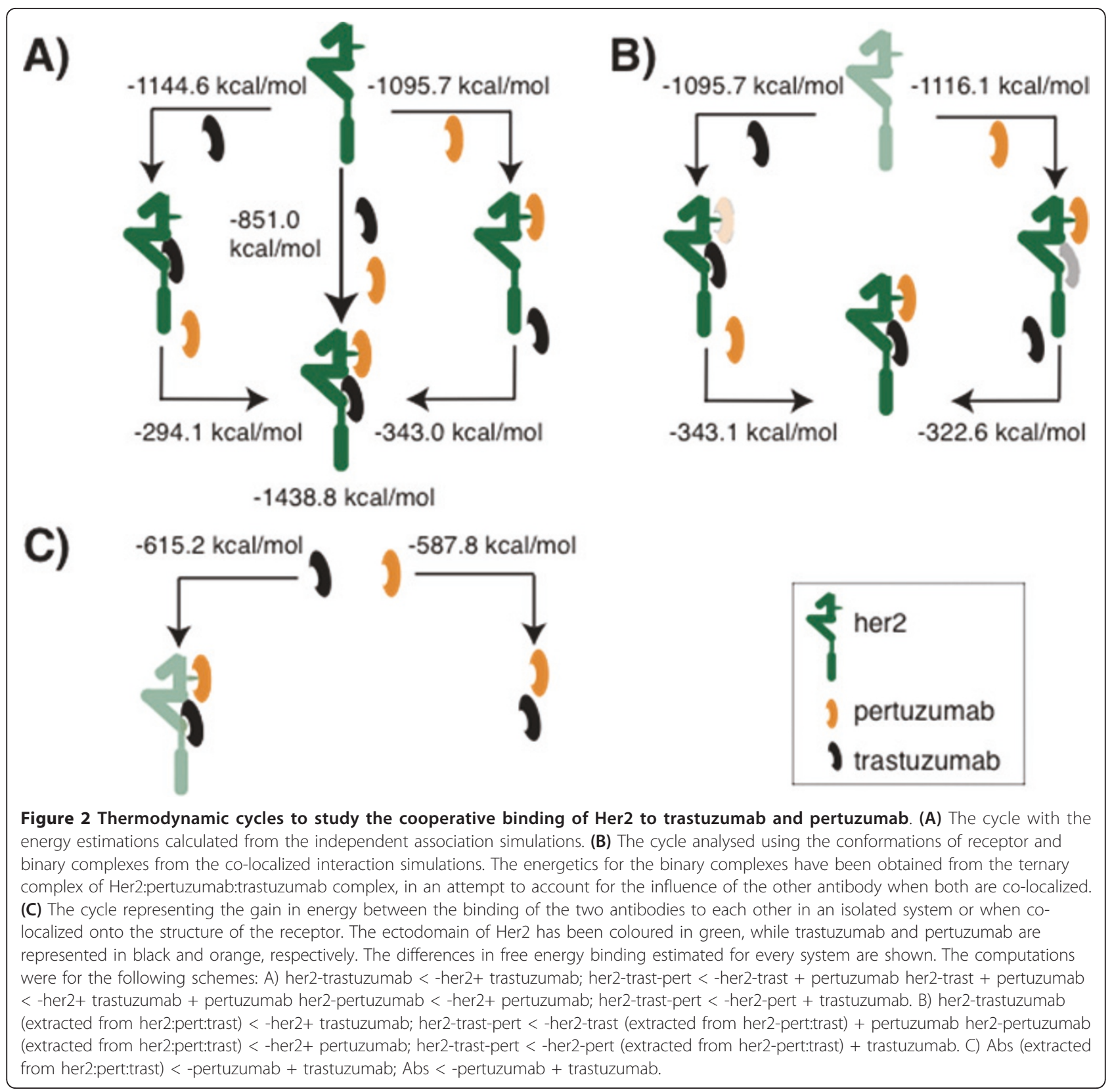

of approximately $50^{\circ}$ for the parallel and approximately $78^{\circ}$ for the motion perpendicular to the membrane), which represents periscope-like movements that this receptor might execute in search of binding partners. In the case of the ternary complex, the binding of trastuzumab causes a shift in the hinge region along domain IV. This is associated with small swivelling motions across an axis orthogonal to the membrane; if the receptor lies parallel to the membrane [34] then this would represent "out of the membrane" motions. The dimerization arm spans approximately $16 \AA$ in comparison to the apo form which covers approximately $33 \AA$ (measured from the $\mathrm{C} \alpha$ of the atom (Pro252) at the base of this motif). In contrast, in the binary states, the pattern of motion differs: domain IV behaves as a rigid body and only one hinge region is apparent. When trastuzumab is bound, a hinge motion is observed $\left(18^{\circ}\right)$ between the bulky domain I-to-III and domain IV (crystallographic data show approximately $7^{\circ}$ in the angle subtended between domains III and IV); the Her2:pertuzumab complex undergoes more complex motions. The deletion in the truncated mutant results in attenuation of two-body rotations along the parallel axis (from $48^{\circ}$ in the "full-length" receptor to approximately $28^{\circ}$ in the truncated form). In contrast, the bound form of this truncated receptor undergoes a hinged motion spanning approximately $20^{\circ}$. 


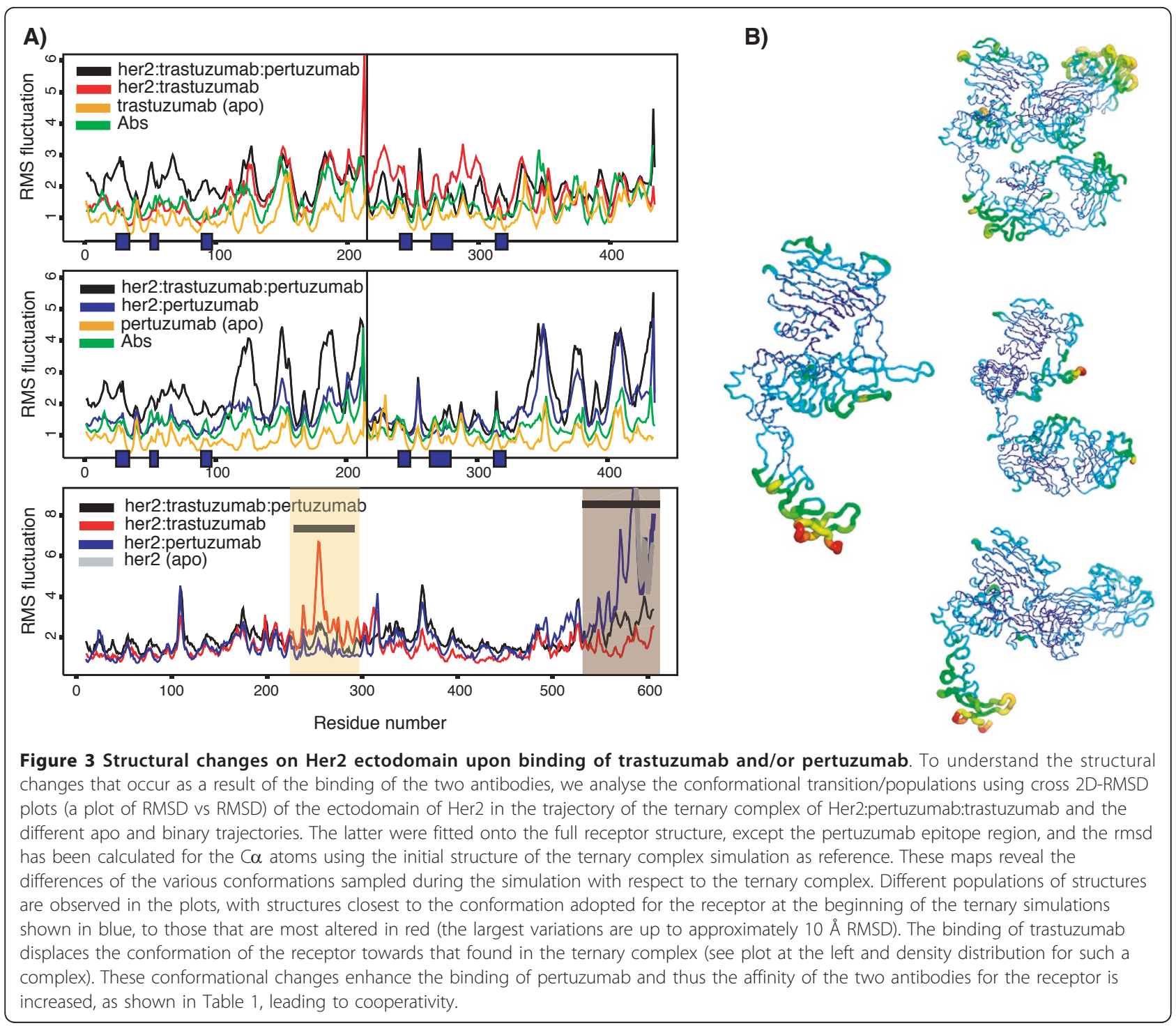

The overall comparison of the conformations that Her2 ectodomain adopts in the different conditions analysed here indicates that domains I, II and III adopt a relatively fixed interdomain relationship, but their relative orientations with respect to domain IV varies considerably (up to approximately $80^{\circ}$ ), and appear to be pivoted across the region 504 to 532 that lies between domains III and IV. This is indicative of the plasticity inherent in these receptors and suggests that the extracellular domain can swivel quite considerably across domain IV that itself is constrained by being embedded partly in the membrane.

\section{Conclusions}

In summary, MD simulations suggest that the clinical synergism observed for the two antibodies arises partly from an enhanced affinity that originates in cooperative interactions between these two antibodies when they are co-localized on Her2 and "clamp" it; this may inhibit dimerization and possibly higher oligomerizations with neighbouring receptors. In the presence of trastuzumab, the receptor becomes highly plastic, especially domains I-III, and this appears to promote increased association with pertuzumab. While combining antibodies is a feature that increasingly appears to have major effects in therapy (see, for example, the work of Chao and coworkers [35], which combines two different antibodies against two different cell surface molecules for treatment of non-Hodgkin's lymphoma), a model incorporating the idea that two antibodies bind to the same receptor, albeit at distinct epitopes, is relatively new; indeed there has been only one report of such a feature as highlighted for targeting the IGF-1 receptor [13]. These findings could be tested, for example, in 2D and 
3D preclinical cell culture models to explore trastuzumab efficacy $[36,37]$. While we await experimental investigations of this novel mechanism in Her2, if validated, it offers routes towards the design of new antibodies whereby combinations of trastuzumab and pertuzumab (or parts thereof) may be constructed that have the potential to generate therapeutics with much higher affinities and hopefully efficacies. The technology of generating hybrid antibodies or bi-specific antibodies [38] is known; however, this technique has been used for linking two antibodies that target different molecules. In contrast our hypotheses open the avenue for linking two antibodies that target distinct epitopes on the same molecule.

\section{Additional material}

Additional file 1: Supplemental Figure S1. Cartoon representation of all the systems involved in the study; A) Apo Her2; B) binary complex between Her2:pertuzumab; C) binary complex between Her2: trastuzumab; D) ternary complex between Her2:pertuzumab:trastuzumab; E) pertuzumab; F) trastuzumab; G) "binary" interaction between trastuzumab:pertuzumab. Trastuzumab has been coloured in black; while pertuzumab is shown in orange.

Additional file 2: Supplemental Figure S2. The characterization of the collective protein dynamics have been analyzed using Principal Component Analysis (PCA). PCA is a statistical method that highlight similarities and differences in a complex data set by reducing the number of dimensions without losing much of the information. In MD simulations, the application of PCA can separate the configurational space into two subspaces; the essential one containing the functionally important correlated motions (comprising only few degrees of freedom or eigenvectors) and the irrelevant subspace with the independent Gaussian fluctuations with little functional relevance. In this study, PCA has been performed on an ensemble of conformations derived from the different MD simulations. Results of PCA obtained from the diagonalization of the atomic displacement correlation matrix of $\mathrm{C} \alpha$ atoms of the ectodomain from six of the MD simulations carried out in this study (using standard Euclidean distances) are shown here. The resulting Principal Components (PCs) are sorted according to their contribution to the total fluctuation along the ensemble of conformations, and only a small subset of these PCs are necessary to describe the great majority of the total atomic displacements which capture the essential dynamics of the conformational changes occurring in the system (the above so-referred essential subspace). Every figure contains the percentage of the cumulative eigenvalues as a function of the number of eigenvectors considered. The inset includes the projection on the first two eigenvectors of the coordinates extracted from the time interval of 8 to 16 ns. Every figure represents the coordinates for the ectodomain of Her2 from the following simulations: (A) apo Her2; (B) Her2:pertuzumab:trastuzumab; (C) Her2:pertuzumab; (D) Her2:trastuzumab; (E) apo truncated Her2; (F) truncated Her2: pertuzumab:trastuzumab. The application of PCA on data obtained from MD simulations provides a useful way of detecting global, correlated motions of the system that are separate from the tightly-constrained harmonic motions of more constrained atoms. The structures projected on the first eigenvectors have facilitated our understanding of the dynamics of the six systems analysed here (see Supplementary Figure S6).

Additional file 3: Supplemental Figure S3. C $\alpha$ RMS deviation with respect to the starting frame of the 2 -ns equilibration phase versus time. (A) apo Her2 simulation; (B) ternary complex Her2:trastuzumab: pertuzumab; (C) binary complex Her2:trastuzumab; (D) binary complex Her2:pertuzumab. The top panel for all the plots represent the RMSD for the whole system; this is broken into the different components in the association in the bottom plots.

Additional file 4: Supplemental Figure S4. RSM fluctuation plots and cartoon figures for the different systems of Her2 in the apo-and holo-states with pertuzumab and trastuzumab. A) RMS fluctuations for trastuzumab-containing systems (top); RMS fluctuations for pertuzumab-containing systems (middle panel) and RMS fluctuations for the ectodomain of the receptor in the four different MD simulations; B) putty/sausage cartoon representation for apo-receptor (right), Her2 in complex with the two antibodies, with trastuzumab and with pertuzumab (from top to bottom) and colored accordingly to the fluctuation values per residue. The complementarity determining regions (CDR) of both antibodies have been marked in the plot with blue boxes. The trastuzumab and pertuzumab epitopes have been highlighted in shaded boxes in grey and cream and black, respectively.

Additional file 5: Supplemental Table S1. $\mathrm{C} \alpha-\mathrm{C} \alpha$ distances for the residues at the receptor:Ab interfaces detected along the simulations between Her2 and the two Abs: pertuzumab and trastuzumab. The different shading boxes indicate the CDR of the antibodies.

Additional file 6: Supplemental Figure S5. Interactions between the two antibodies and new epitope revealed in the MD simulations (A) Density distributions for the intermolecular $\mathrm{C} \alpha-\mathrm{C} \alpha$ distance between Glu294 and Asp295 (labeled in the Figure C) in the pertuzumab-induced trastuzumab epitope of the receptor and their putative interacting residues at the interface in trastuzumab. The dashed lines represent the population in the Her2:pertuzumab:trastuzumab simulation, while the straight lines show the distribution found in the Her2:trastuzumab simulation. (B) Density distribution for the intermolecular $\mathrm{C} \alpha-\mathrm{C} \alpha$ distance between residues in the different Abs. In horizontal lines the average distance for the ternary (red) and binary (blue) simulations are shown. (C) Schematic representation of the residues for which the distances have been measured. The color coding is the same as in Figure S1.

Additional file 7: Supplemental Figure S6. Cartoon representation of the maximum and minimum conformations of Her2 along the first eigenvector extracted from the PCA analysis and colored according to the Dyndom output (see Additional file 8) for the following simulations: (A) apo Her2; (B) Her2:pertuzumab:trastuzumab; (C) Her2: pertuzumab; (D) Her2:trastuzumab; (E) apo truncated Her2; (F) truncated Her2:pertuzumab:trastuzumab. All the models were fit onto the last 20 residues of the ectodomain.

Additional file 8: Supplemental Table S2. Dynamical domains and hinge bending residues determined by the program DYNDOM for the superposition of the Her2 receptor of the trajectories of this domain in the apo, dimeric and trimeric forms with trastuzumab and pertuzumab.

\section{Abbreviations}

$\Delta G_{\text {bind: }}$ : binding free energy; $\Delta G_{\mathrm{ELE}}$ : electrostatics energy; $\Delta G_{\mathrm{NP}}$ : non-polar energy; $\triangle G_{V D}$ : van der Waals energy; Abs: antibodies; CDR: complementary determining regions; EGFR: epidermal growth factor receptor; Fab: fragment antigen binding; GB: Generalized-Born; HER2: human epidermal growth factor 2; IGF-1: insulin-like growth factor; LCPO: linear combination of pairwise overlaps; MD: molecular dynamics; MM/GBSA: molecular mechanics/ Generalized-Born surface Area; PCA: Principal component analysis; PME: particle mesh Ewald; RTK: receptor tyrosine kinase; SASA: solvent-accessible surface area;

\section{Acknowledgements}

CSV is adjunct at DBS (NUS) and SBS (NTU): Grant from European Research Council (AdG09 250244) is acknowledged.

\section{Author details}

'Bioinformatics Institute (A*STAR), 30 Biopolis Street, \#07-01 Matrix, 38671, Singapore. ${ }^{2}$ Division of Hematology and Oncology, Massachusetts General Hospital Cancer Center, Harvard Medical School, 10 North Grove Street, LH108, Boston, MA 02114, USA. ${ }^{3}$ Medical Oncology Department, Vall d'Hebron Institute of Oncology (VHIO), Vall d'Hebron University Hospital, Passeig Vall 
d'Hebron 119-129, Barcelona 08035, Spain. ${ }^{4}$ Department of Biological Sciences, National University of Singapore, 14 Science Drive 4, 117543, Singapore. ${ }^{5}$ School of Biological Sciences, Nanyang Technological University, 60 Nanyang Drive, 637551, Singapore.

\section{Authors' contributions}

GF conceived of the study, participated in study design and data interpretation, and drafted the manuscript. MS and JB conceived of the study and revised the manuscript critically for important intellectual content. CV conceived of the study, participated in the analysis and interpretation, and helped to draft the manuscript. All authors read and approved the final manuscript.

\section{Competing interests}

The authors declare that they have no competing interests.

Received: 22 November 2010 Revised: 21 March 2011

Accepted: 22 May 2011 Published: 22 May 2011

\section{References}

1. Yarden Y, Sliwkowski MX: Untangling the ErbB signalling network. Nat Rev Mol Cell Biol 2001, 2:127-137.

2. Holbro T, Hynes NE: ErbB receptors: directing key signaling networks throughout life. Annu Rev Pharmacol Toxicol 2004, 44:195-217.

3. Cho HS, Mason K, Ramyar KX, Stanley AM, Gabelli SB, Denney DW, Leahy DJ: Structure of the extracellular region of Her2 alone and in complex with the Herceptin Fab. Nature 2003, 421:756-60.

4. Garrett TP, McKern NM, Lou M, Elleman TC, Adams TE, Lovrecz GO, Kofler M, Jorissen RN, Nice EC, Burgess AW, Ward CW: The crystal structure of a truncated ErbB2 ectodomain reveals an active conformation, poised to interact with other ErbB receptors. Mol Cell 2003, 11:495-505.

5. Jorissen RN, Walker F, Pouliot N, Garrett TP, Ward CW, Burgess AW: Epidermal growth factor receptor: mechanisms of activation and signalling. Exp Cell Res 2003, 284:31-53.

6. Schlessinger J: Cell signaling by receptor tyrosine kinases. Cell 2000, 103:211-225.

7. Slamon DJ, Godolphin W, Jones LA, Holt JA, Wong SG, Keith DE, Levin WJ, Stuart SG, Udove J, Ullrich A: Studies of the HER-2/neu proto-oncogene in human breast and ovarian cancer. Science 1989, 244:707-712.

8. Slamon DJ: Proto-oncogenes and human cancers. N Engl J Med 1987, 317:955-957.

9. Baselga J, Swain SM: Novel anticancer targets: revisiting ERBB2 and discovering ERBB3. Nat Rev Cancer 2009, 9:463-475.

10. Harari D, Yarden Y: Molecular mechanisms underlying ErbB2/Her2 action in breast cancer. Oncogene 2000, 19:6102-6114.

11. Sliwkowski MX, Lofgren JA, Lewis GD, Hotaling TE, Fendly BM, Fox JA: Nonclinical studies addressing the mechanism of action of trastuzumab (Herceptin). Semin Oncol 1999, 26:60-70.

12. Baselga J, Gelmon KA, Verma S, Wardley A, Conte P, Miles D, Bianchi G, Cortes J, McNally VA, Ross GA, Fumoleau P, Gianni L: Phase II trial of pertuzumab and trastuzumab in patients with human epidermal growth factor receptor 2-positive metastatic breast cancer that progressed during prior trastuzumab therapy. J Clin Oncol 2010, 28:1138-1144.

13. Dong J, Demarest SJ, Sereno A, Tamraz S, Langley E, Doern A, Snipas T, Perron K, Joseph I, Glaser SM, Ho SN, Reff ME, Hariharan K: Combination of two insulin-like growth factor-I receptor inhibitory antibodies targeting distinct epitopes leads to an enhanced antitumor response. Mol Cancer Ther 2010, 9:2593-2604.

14. Franklin MC, Carey KD, Vajdos FF, Leahy DJ, de Vos AM, Sliwkowski MX: Insights into ErbB signaling from the structure of the ErbB2-pertuzumab complex. Cancer Cell 2004, 5:317-328.

15. Case DA, Darden TA, Cheatham TE III, Simmerling CL, Wang J, Duke RE, Luo R, Merz KM, Pearlman DA, Crowley M, Walker RC, Zhang W, Wang B, Havik S, Riotberg A, Seabra G, Wong KF, Paesani F, Wu X, Brozell S, Tsui V, Gohlke H, Yang L, Tan C, Mongan J, Hornak V, Cui G, Beroza P, Mathews DH, Schafmeister C, et al: AMBER 9. University of California, San Francisco; 2006.

16. Wang J, Cieplak P, Kollman PA: How well does a restrained electrostatic potential (RESP) model perform in calculating conformational energies of organic and biological molecules? J Comput Chem 2000, 21:1049-1074.
17. Dolinsky TJ, Nielsen JE, McCammon JA, Baker NA: PDB2PQR: an automated pipeline for the setup of Poisson-Boltzmann electrostatics calculations. Nucleic Acids Res 2004, 32:W665.

18. Izaguirre JA, Catarello DP, Wozniak JM, Skeel RD: Langevin stabilization of molecular dynamics. J Chem Phys 2001, 114:2090.

19. Berendsen HJC, Postma JPM, Van Gunsteren WF, DiNola A, Haak JR: Molecular dynamics with coupling to an external bath. J Chem Phys 1984, 81:3684

20. Darden T, York D, Pedersen L: Particle mesh Ewald: An N log $(\mathrm{N})$ method for Ewald sums in large systems. J Chem Phys 1993, 98:10089-10092.

21. Ryckaert JP, Ciccotti G, Berendsen HJC: Numerical integration of the Cartesian equations of motion of a system with constraints: molecular dynamics of n-alkanes. J Comput Phys 1977, 22:327-341.

22. Grant BJ, Rodrigues APC, ElSawy KM, McCammon JA, Caves LSD: Bio 3 d: an $\mathrm{R}$ package for the comparative analysis of protein structures. Bioinformatics 2006, 22:2695.

23. Hayward S, Berendsen HJC: Systematic analysis of domain motions in proteins from conformational change: new results on citrate synthase and T4 lysozyme. Proteins 1998, 30:144-154.

24. PyMOL. DeLano Scientific, Palo Alto, CA; [http://www.pymol.org].

25. Kollman PA, Massova I, Reyes C, Kuhn B, Huo S, Chong L, Lee M, Lee T, Duan Y, Wang W, Donini O, Cieplak P, Srinivasan J, Case DA, Cheatham TE: Calculating structures and free energies of complex molecules: combining molecular mechanics and continuum models. Acc Chem Res 2000, 33:889-897.

26. Weiser J, Shenkin PS, Still WC: Approximate atomic surfaces from linear combinations of pairwise overlaps (LCPO). J Comput Chem 1999, 20:217-230.

27. Amadei $A$, Linssen $A B$, Berendsen $H J$ : Essential dynamics of proteins. Proteins 1993, 17:412-425.

28. Levy RM, Srinivasan AR, Olson WK, McCammon JA: Quasi-harmonic method for studying very low frequency modes in proteins. Biopolymers 1984, 23:1099-1112.

29. Stepanova M: Dynamics of essential collective motions in proteins: theory. Phys Rev E 2007, 76:51918.

30. Gohlke H, Kiel C, Case DA: Insights into protein-protein binding by binding free energy calculation and free energy decomposition for the Ras-Raf and Ras-RalGDS complexes. J Mol Biol 2003, 330:891-913.

31. Balius TE, Rizzo RC: Quantitative Prediction of Fold Resistance for Inhibitors of EGFR. Biochemistry 2009, 48:8435-8448.

32. McGillick BE, Balius TE, Mukherjee S, Rizzo RC: Origins of Resistance to the HIVgp41 Viral Entry Inhibitor T20. Biochemistry 2010, 49:3575-3579.

33. Troise F, Cafaro V, Giancola C, D'Alessio G, De Lorenzo C: Differential binding of human immunoagents and Herceptin to the ErbB2 receptor. FEBS J 2008, 275:4967-4979.

34. Kästner J, Loeffler HH, Roberts SK, Martin-Fernandez ML, Winn MD: Ectodomain orientation, conformational plasticity and oligomerization of ErbB1 receptors investigated by molecular dynamics. J Struct Biol 2009, 167:117-128

35. Chao MP, Alizadeh AA, Tang C, Myklebust JH, Varghese B, Gill S, Jan M, Cha AC, Chan CK, Tan BT, Park CY, Zhao F, Kohrt HE, Malumbres R, Briones J, Gascoyne RD, Lossos IS, Levy R, Weissman IL, Majeti R: Anti-CD47 antibody synergizes with rituximab to promote phagocytosis and eradicate non-Hodgkin lymphoma. Cell 2010, 142:699-713.

36. Pickl M, Ries $\mathrm{CH}$ : Comparison of $3 \mathrm{D}$ and $2 \mathrm{D}$ tumor models reveals enhanced HER2 activation in 3D associated with an increased response to trastuzumab. Oncogene 2009, 28:461-468

37. Weigelt B, Lo AT, Park CC, Gray JW, Bissell MJ: HER2 signaling pathway activation and response of breast cancer cells to HER2-targeting agents is dependent strongly on the 3D microenvironment. Breast Cancer Res Treat 2010, 122:35-43.

38. Thakur A, Lum LG: Cancer therapy with bispecific antibodies: Clinical experience. Curr Opin Mol Ther 2010, 12:340-349.

\section{doi:10.1186/bcr2888}

Cite this article as: Fuentes et al: Synergy between trastuzumab and pertuzumab for human epidermal growth factor 2 (Her2) from colocalization: an in silico based mechanism. Breast Cancer Research 2011 13:R54. 